\title{
Cancer Survivors Aged 40 Years or Elder are Associated with High Risk of Chronic Kidney Disease: The 2010-2012 Korean National Health and Nutrition Examination Survey
}

\author{
Hyun-Young Shin ${ }^{1}$, John A Linton ${ }^{1,2}$, Jae-Yong Shim ${ }^{3}$, Hee-Taik Kang ${ }^{3,4 *}$
}

\begin{abstract}
Background: The number of cancer survivors is increasing globally and recently, higher rates of comorbidities in cancer survivors have been reported. However, no studies have investigated whether cancer survivors have a higher risk of chronic kidney disease (CKD). Accordingly, our study evaluated the association between cancer survivors and the risk of CKD using the 2010-2012 Korean National Health and Nutrition Examination Survey. Materials and Methods: A total of 11,407 participants aged 40 years and over were categorized into two groups according to cancer experience. Multiple variables were compared and the odds ratios (ORs) for CKD prevalence were calculated using a weighted logistic regression analysis between the two groups. Results: Cancer survivors were older than were those in the non-cancer group, on average, the percentages of glomerular filtration rate(GFR) lower than $60 \mathrm{~mL} / \mathrm{min} / 1.73 \mathrm{~m}^{2}$, proteinuria, and CKD were significantly higher in cancer survivors when compared to controls. Weighted logistic regression analyses demonstrated that cancer survivors had a higher risk for CKD after adjusting for multiple variables (OR (95\% confidence interval), 2.88 (1.485.59)). Conclusions: Our study demonstrated a possible association between CKD and cancer survival in Korean adults. Identifying and correcting risk factors for cancer survivors would positively affect prevention of CKD and result in a better cancer prognosis.
\end{abstract}

Keywords: Cancer survivor - chronic kidney disease - glomerular filtration rate - proteinuria

Asian Pac J Cancer Prev, 16 (4), 1355-1360

\section{Introduction}

Cancer survivors are defined as "individuals who have experienced any kind of malignancies and are still alive" (Denlinger et al., 2014). The number of cancer survivors is increasing globally, with approximately 13.7 million in the US (Centers for Disease, et al., 2011) and one million in Korea. In Korea, there were a reported 426,824 cancer survivors over 65 years old in 2012. This might be due to a range of factors, including earlier detection of cancer, improved cancer treatments, and increased national public health concern (Denlinger et al., 2014). Recently, higher rates of comorbidities in cancer survivors than in the general population have been reported. The presence of comorbidities, such as cardiovascular, cerebrovascular, and other chronic diseases including hypertension and diabetes, could threaten cancer survivors' life expectancy and quality of life (Ogle et al., 2000). As a result of these increased risks, it is necessary for public health authorities to pay special attention to the care of cancer survivors.

Chronic kidney disease is a global epidemic disease. The global trend towards an aged population is related to increasing CKD prevalence. The burden of CKD on public health is increasing in severity (Jha et al., 2013). Modifiable lifestyle behaviors, such as smoking cigarettes, drinking alcohol, and physical inactivity, are well-known causes of CKD, in addition to aging. CKD shares these risk factors with cancers and cardiovascular diseases, which are the main causes of death among cancer survivors (Foundation, 2002; Levey et al., 2005; White et al., 2009; Stump, 2011). Several studies have reported that patients with renal impairment are more susceptible to developing cancer (Maisonneuve et al., 1999; Cengiz et al., 2002). However, no studies have investigated whether cancer survivors are at a higher risk of developing CKD. Accordingly, we aimed to evaluate the association between cancer survival and risk of CKD using the 2010-2012 Korean National Health and Nutrition Examination Survey.

\section{Materials and Methods}

Study population

The Korean National Health and Nutrition Examination

${ }^{1}$ Department of Family Medicine, ${ }^{2}$ International Health Care Center, Severance Hospital, ${ }^{3}$ Department of Family Medicine, Gangnam Severance Hospital, Yonsei University College of Medicine, ${ }^{4}$ Department of Medicine, Graduate School, Yonsei University, Seoul, Republic of Korea*For correspondence: KANGHT@yuhs.ac 
Survey is a nationwide survey representing the general Korean population that is conducted by the Korean Ministry of Health and Welfare. It includes comprehensive information on health status, health behavior, and sociodemographics. The sampling units were households selected using a stratified, multistage, probability-sampling design according to geographic area, sex, and age group based on household registries. Participants were asked to complete four parts of a questionnaire: a health interview survey, health behavior survey, health examination survey, and nutrition survey. The health interview survey was conducted through face-to-face interviews at participants' homes by trained interviewers. Citizens had the right to refuse participation according to the National Health Enhancement Act. Participants who agreed to participate provided written informed consent. The Korea Centers for Disease Control and Prevention obtained participants' agreement to use blood samples taken during the health interview survey for further research.

This study was based on data from 19,394 adults in 192 national districts obtained from the Korean National Health and Nutrition Examination Survey V (2010-2012). We excluded participants younger than 40 years $(n=4.474)$, those who had incomplete laboratory data $(\mathrm{N}=3.231)$, and those who did not have information regarding cancer diagnosis $(\mathrm{N}=280)$. After these exclusions, 11,409 were included in the final analysis. The Institutional Review Board of the Korea Centers for Disease Control and Prevention approved this study.

\section{Measurement of anthropometry and laboratory data}

Trained medical staff performed the physical examinations by following standardized procedures. Body weight and height were measured to the nearest 0.1 $\mathrm{kg}$ and $0.1 \mathrm{~cm}$, respectively, with subjects wearing light indoor clothing without shoes. Body mass index (BMI) was calculated as the ratio of weight in kilograms to height in squared meters. Blood pressure was measured twice on the right arm at 5-minute intervals using a standard mercury sphygmomanometer (Baumanometer; Baum, Copiague, NY, USA) and recorded as an averaged value. After overnight fasting, blood samples were obtained from participants' antecubital veins. Levels of fasting plasma glucose, total cholesterol, and creatinine were measured enzymatically using a Hitachi Automatic Analyzer 7600 (Hitachi, Japan). Dipstick urinalyses were conducted with an Urisys 2400 (Roche, Germany).

\section{Definition of cancer survivor, chronic kidney disease, and health-related lifestyle factors}

Participants who answered "Yes" to "Have you ever been diagnosed with any kind of cancer or malignancy in your life?" were classified as cancer survivors. Those who answered "No" were the control group.

An estimated GFR was calculated using the abbreviated equation from the Modification of Diet in Renal Disease (MDRD) study: estimated GFR (mL/min/1.73 $\left.\mathrm{m}^{2}\right)=186.3 \times\left(\right.$ serum creatinine $\left.\mathrm{e}^{-1.154}\right) \times\left(\right.$ age $\left.^{-0.203}\right) \times 0.742$ (if female). CKD was defined based on either renal tissue damage or reduced renal function. CKD was defined as estimated GFR $<60 \mathrm{~mL} / \mathrm{min} / 1.73 \mathrm{~m}^{2}$ or proteinuria 1+ or greater. Proteinuria was tested using dipstick methods with freshly voided urine samples.

Information on health-related lifestyles was obtained from data gathered from the self-report questionnaire during the interview portion of each survey period. Individuals who engaged in $\geq 20$ min of vigorousintensity physical activity at least three days a week or $\geq 30$ min of light- to moderate-intensity physical activity at least five days a week were categorized as the regular exercise group using the short form of the International Physical Activity Questionnaire (IPAQ). To identify high-risk drinkers, we used the Alcohol Use Disorders Identification Test (AUDIT), which consists of three domains: hazardous alcohol use, dependence symptoms, and harmful alcohol use (Thomas et al., 2001). The cutoff points of the AUDIT score were categorized into three groups: low-risk drinkers, 0-7; intermediate-risk drinkers, 8-14; and high-risk drinkers, $\geq 15$ points. Individuals who smoked cigarettes during the relevant survey period were categorized as current smokers. Daily calorie intake was monitored by a 24 -h food recall and analyzed using CAN-Pro 3.0 software (Korean Nutrition Society, Seoul, Korea). The prevalence of diabetes mellitus was calculated using the number of people who answered "yes" to either of the following questions: "have you ever been diagnosed with diabetes mellitus?" or "are you currently taking any medication for diabetes mellitus?" Similarly, the prevalence of hypertension was calculated using the number of people who answered "yes" to either of the following questions: "have you ever been diagnosed with hypertension?" or "are you currently taking any medication for hypertension?"

\section{Statistical analysis}

Data taken from the Korea National Statistical Office was used to define the standard population. In order to represent the entire population of Korean adults without biased estimates, sampling weights were used to account for the complex sampling. All data from continuous variables are presented as means and standard errors (SEs). Data from categorical variables are presented as percentages. Student t-tests and chi-squared tests were used to compare mean values for continuous variables and percentages for categorical variables, respectively, by cancer survival status. The odds ratios (ORs) and corresponding $95 \%$ confidence intervals $(95 \% \mathrm{CI})$ for risk of CKD were calculated using weighted multivariate logistic regression analyses. All analyses were conducted using SAS statistical software, version 9.2 (SAS Institute Inc, Cary, NC). All statistical tests were two-tailed and statistical significance was determined as $\mathrm{p}<0.05$.

\section{Results}

The characteristics of the study participants are summarized in Table 1 . The mean age was 55.8 years old and the rate of cancer survivors was $5.3 \%$. The mean value of GFR and creatinine levels were $90.5 \mathrm{~mL} / \mathrm{min} / 1.73$ $\mathrm{m}^{2}$ and $0.85 \mathrm{mg} / \mathrm{dL}$, respectively. Cancer survivors were significantly older than were those in the non-cancer group, and a mean of 18.5 years had passed since cancer 
Table 1. Basic Characteristics of the Study Subjects

\begin{tabular}{|c|c|c|c|c|}
\hline & Total & Non-cancer & Cancer Survivor & p-value ${ }^{a}$ \\
\hline Unweighted $\mathrm{N}$ (Female, \%) & $11.409(52.2)$ & $10727(51.6)$ & $682(63.8)$ & \\
\hline Age (years) & $55.8 \pm 0.2$ & $55.6 \pm 0.2$ & $59.8 \pm 0.6$ & $<0.001$ \\
\hline Time since cancer diagnosis (years) & NA & NA & $18.5 \pm 1.1$ & NA \\
\hline Body mass index $\left(\mathrm{kg} / \mathrm{m}^{2}\right)$ & $24.0 \pm 0.0$ & $24.1 \pm 0.0$ & $23.6 \pm 0.2$ & 0.006 \\
\hline Systolic blood pressure (mmHg) & $123.1 \pm 0.3$ & $123.1 \pm 0.3$ & $123.2 \pm 0.8$ & 0.861 \\
\hline Diastolic blood pressure $(\mathrm{mmHg})$ & $78.1 \pm 0.2$ & $78.1 \pm 0.2$ & $76.5 \pm 0.5$ & 0.001 \\
\hline Fasting plasma glucose $(\mathrm{mg} / \mathrm{dL})$ & $101.0 \pm 0.3$ & $100.9 \pm 0.3$ & $101.7 \pm 1.1$ & 0.503 \\
\hline Total cholesterol (mg/dL) & $193.9 \pm 0.5$ & $194.0 \pm 0.5$ & $192.2 \pm 1.8$ & 0.326 \\
\hline Creatinine $(\mathrm{mg} / \mathrm{dL})$ & $0.85 \pm 0.00$ & $0.85 \pm 0.00$ & $0.84 \pm 0.01$ & 0.504 \\
\hline GFR $\left(\mathrm{mL} / \mathrm{min} / 1.73 \mathrm{~m}^{2}\right)$ & $90.5 \pm 0.3$ & $90.7 \pm 0.3$ & $87.5 \pm 0.8$ & $<0.001$ \\
\hline Calorie intake (Kcal/day) & $1986.0 \pm 12.6$ & $1998 \pm 13$ & $1765 \pm 36$ & $<0.001$ \\
\hline Household income (Korean Won/month) & $4291 \pm 159$ & $4338 \pm 167$ & $3434 \pm 358$ & 0.023 \\
\hline Regular exerciser $(\%)^{\mathrm{b}}$ & 45 & 45.4 & 38.8 & 0.006 \\
\hline High-risk drinker $(\%)^{\mathrm{b}}$ & 16.1 & 16.3 & 10.2 & 0.018 \\
\hline Current smoker $(\%)^{\mathrm{b}}$ & 25.8 & 26.7 & 9.4 & $<0.001$ \\
\hline Prevalence of diabetes $(\%)^{\mathrm{b}}$ & 8.6 & 10 & 12.7 & $<0.001$ \\
\hline Prevalence of hypertension $(\%)^{\mathrm{b}}$ & 50.9 & 54 & 45.9 & 0.483 \\
\hline
\end{tabular}

*All data except regular exerciser, high-risk drinker, current smoker, prevalence of diabetes and prevalence of hypertension are represented as mean $\pm \mathrm{SE}$; ${ }^{\mathrm{p}} \mathrm{p}$-values are determined by student $\mathrm{t}$-tests between non-cancer and cancer survivor groups; ${ }^{\mathrm{b}} \mathrm{Regular}$ exerciser, high-risk drinker, current smoker, prevalence of diabetes and prevalence of hypertension are represented as percentage and the p-values are determined by chi-square tests. 1 USD=1000 Korean Won; Regular exerciser: A person who incorporates $\geq 20$ min of vigorous-intensity physical activity for $\geq 3$ days a week, or light-/moderate-intensity physical activity $\geq 5$ days a week High-risk drinker: AUDIT score $\geq 15$ Abbreviations: GFR, glomerular filtration rate

survivors were initially diagnosed. Mean BMI, diastolic blood pressure, daily calorie intake, and monthly household income were significantly lower in cancer survivors than in the non-cancer group. The mean GFR was lower in cancer survivors (87.5 in cancer survivors vs $90.7 \mathrm{~mL} / \mathrm{min} / 1.73 \mathrm{~m}^{2}$ in the non-cancer group), while mean levels of creatinine were not significantly different between the two groups. The percentages of regular exercisers, high-risk drinkers, and current smokers were lower in cancer survivors. The prevalence of diabetes mellitus was higher in cancer survivors; however, the prevalence of hypertension did not significantly differ between the two groups.

Figure 1 shows GFR, the prevalence of proteinuria and CKD by cancer experience. There was a significant difference $(p<0.001)$ between the percentage of participants with GFR lower than $60 \mathrm{~mL} / \mathrm{min} / 1.73 \mathrm{~m}^{2}$ between cancer survivors $(6.2 \%)$ and the non-cancer group $(3.0 \%)$. Although the prevalence of proteinuria was not significantly different between groups $(2.2 \%$ in cancer survivors and $1.4 \%$ in the non-cancer group; $\mathrm{p}=0.280$ ), prevalence of CKD was significantly different (7.4\% in cancer survivors compared to $4 \%$ in the non-cancer group; $\mathrm{p}=0.001)$. We performed weighted logistic regression analyses to examine whether cancer survivors demonstrated a higher risk for CKD (Table $2)$. In comparison with the non-cancer group, age and sex-adjusted ORs (95\%CIs) of cancer survivors for CKD were $1.48(0.98-2.23)$ in Model 1. After adjusting for age, sex, systolic blood pressure, BMI, fasting plasma glucose, total cholesterol, daily calorie intake, and monthly household income, ORs (95\% CIs) of cancer survivors for CKD were 1.51 (0.98-2.33) in Model 2. After fully adjusting for lifestyle factors (exercise, smoking, and alcohol consumption) and the potential confounding factors in Model 2, the OR $(95 \% \mathrm{CI})$ were 2.88 (1.485.59) in Model 3.
Table 2. Odds Ratio for Chronic Kidney Disease According to Cancer Experiences

\begin{tabular}{lc}
\hline All & OR (95\%CI) \\
\hline Model 1 & $1.48(0.98-2.23)$ \\
Model 2 & $1.51(0.98-2.33)$ \\
Model 3 & $2.88(1.48-5.59)$ \\
\hline
\end{tabular}

*Model 1: adjusted for age and sex; Model 2: adjusted for age, sex, systolic blood pressure, body mass index, fasting plasma glucose, total cholesterol, daily calorie intake, and monthly house income; Model 3: adjusted for age, sex, systolic blood pressure, body mass index, fasting plasma glucose, total cholesterol, daily calorie intake, monthly house income, exercise status (Regular exerciser: $\geq 20 \mathrm{~min}$ of vigorous-intensity physical activity for $\geq 3$ days a week, or light-/moderate-intensity physical activity $\geq 5$ days a week), smoking status (never smoker, former smoker, current smoker), and drinking status (high-risk drinking: AUDIT score $\geq 15$ )

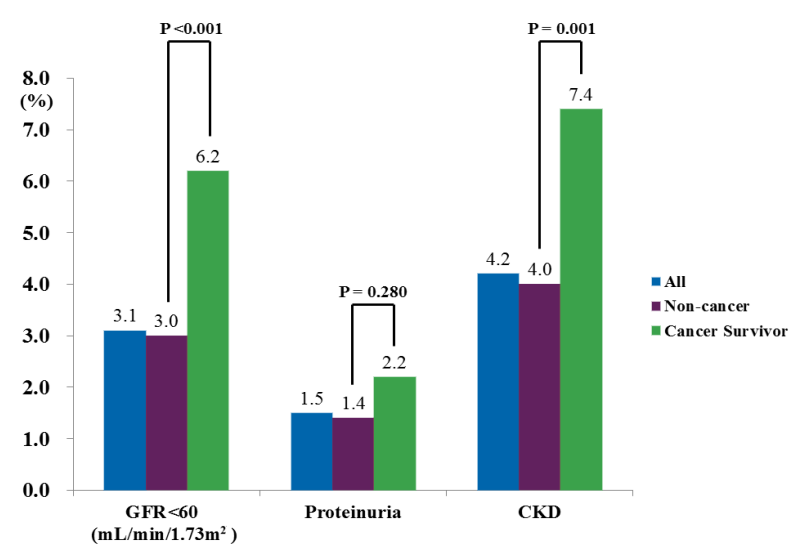

Figure 1. The Percentage of Glomerular Filtration Rate Lesser than $60 \mathrm{~mL} / \mathrm{min} / 1.73 \mathrm{M}^{2}$ and Proteinuria and the Prevalence of Chronic Kidney Disease among Cancer Survivors and Control Group 


\section{Discussion}

In the present study, we found that cancer survivors had a higher risk for CKD after adjusting for possible confounding factors such as age, sex, BMI, blood pressure, and lifestyle (cigarette smoking, alcohol drinking, and exercise status) in this nationally representative sample. Several previous studies have reported the association between CKD and cancer. Many have provided evidence that end-stage renal disease (ESRD) patients have increased risk of developing cancer; this could be explained by immunosuppression linked to uremia and immunosuppressive therapy after transplantation (Maisonneuve et al., 1999; Cengiz et al., 2002; Vajdic et al., 2006). Early-stage CKD has also been associated with a higher risk of cancer incidence (Cengiz et al., 2002; Jorgensen et al., 2008). However, no studies had investigated whether adult cancer survivors are more susceptible to CKD than adults without cancer experience.

One hypothesis that may explain this association between cancer survival and higher risk of CKD is that cancer survivors appear to be more vulnerable to kidney dysfunction and damage because they are typically older and have more comorbid conditions and cancerrelated conditions, such as cachexia and hypercalcemia (Airy et al., 2013). In addition, most survivors have undergone at least one anti-cancer therapy (i.e., surgery, chemotherapy, and radiation treatment). Some treatments, including cisplatin, carboplatin, ifosfamide, radiotherapy, and nephrectomy, are considered nephrotoxic and can cause renal dysfunction, proteinuria, and hypertension (Yao et al., 2007; Dekkers et al., 2013). Dekkers et al. (2013) demonstrated in a retrospective cohort study that adult childhood cancer survivors who were treated with cisplatin, ifosfamide, or abdominal radiation therapy (RT) with nephrectomy are at greater risk for kidney injury. Cisplatin is accumulated and biotransformed into highly reactive thiols in the kidney, thereby leading to kidney injury (Yao et al., 2007). Platinum-containing agents also mediate cytotoxic effects through nuclear and mitochondrial DNA damage and directly induce apoptosis of renal epithelial cells (Siddik et al., 2005). Furthermore, several anticancer agents increase inflammation through the release of inflammatory cytokine and attraction of inflammatory cells, resulting in the pathogenesis of nephrotoxicity (Yao et al., 2007). Renal cell cancer survivors are usually treated with unilateral nephrectomy. Compensatory hypertrophy and hyperfiltration of the remaining kidney after unilateral nephrectomy can have long-term effects such as glomerulosclerosis, proteinuria, and elevated blood pressure (Dekkers et al., 2013). RT can directly damage the kidneys and cause glomerular, tubular, and endothelial microvascular dysfunction in a dose-dependent manner (Quarmby et al., 1999). Acute RT-induced kidney damage is usually subclinical, while chronic RT-induced injury is characterized by elevated blood pressure, serum creatinine levels, and renal failure. The aforementioned anticancer treatments can indirectly cause hypertension and atherosclerosis, which independently affect CKD development. In addition to direct and indirect effects of individual anticancer treatments, other nephrotoxic agents, which are necessary to the development of infectious diseases, could influence the observed higher prevalence of CKD among cancer survivors.

Another possible explanation is that the pathogenesis of CKD shares the risk factors for cancer development. Diabetes and hypertension are the most common causes of CKD (Foundation, 2002; Levey et al., 2005). Both are closely related to modifiable lifestyle factors, such as smoking, drinking, and a sedentary lifestyle (Xin et al., 2001; Levey et al., 2005; Willi et al., 2007; Stump, 2011). Tobacco is known to lead to hypertension, diabetes, and atherosclerosis, as well as CKD (Athyros et al., 2013). Furthermore, it can worsen proteinuria and CKD in diabetic and hypertensive patients. Smoking is also considered a risk factor for several types of carcinoma (Steele et al., 2012). High-risk drinking is associated with renal tubular dysfunction, hypertension, and diabetes, all of which deteriorate kidney function (Steele et al., 2012). In particular, heavy alcohol intake also increases risk for cancers of the digestive system, such as colorectal and liver cancers (Fedirko et al., 2011; Grewal et al., 2012). Previous studies reported that physical activity might play a protective role in the incidence and progression of CKD through the improvement of vascular endothelial function, insulin sensitivity, and control of blood pressure (Johansen, 2007; Stump, 2011). In addition, regular physical exercise can decrease oxidative stress, inflammation, and obesity risk. Accordingly, systematic reviews and meta-analyses have demonstrated that physical activity is inversely associated with cancer risk, including prostate, breast, and colon cancers, despite some debate on these topics (Wolin et al., 2009; Liu et al., 2011; Wu et al., 2013). Taken together, the aforementioned lifestyles could underlie the association between CKD and cancer. Unhealthy lifestyle behaviors, such as smoking, drinking, and physical inactivity, are likely to increase risk for both cancer and renal impairment. For these reasons, cancer survivors might be more vulnerable to CKD before cancer diagnosis. Anticancer treatments might lead to suffering from CKD through the cumulative effects of unhealthy lifestyles.

Several studies have demonstrated that cancer survivors tended to change from unhealthy lifestyles to healthier patterns after cancer diagnosis, including smoking cessation, cutting down on alcohol consumption, increased physical activity, and changing diet patterns (Gritz et al., 1991; Hounshell et al., 2001; Maskarinec et al., 2001; Duffy et al., 2002; Demark-Wahnefried et al., 2005; Sprague et al., 2010). This present study is consistent with those previous studies, showing that cancer survivors pursue healthier behaviors, with a lower percentage of current smokers, heavy drinkers and a lower daily calorie intake compared with the non-cancer group. In addition, cancer survivors had lower BMI and blood pressure. When adjusting for these confounding factors in logistic regression analyses, there appeared to be an effect on the ORs for CKD, which became statistically significant.

This study has several limitations. First, this is a cross-sectional design, so we could not determine the causality of the relationships. Second, The Kidney Disease 
Outcomes Quality Initiative guideline defines CKD as the presence of either renal dysfunction (GFR $<60 \mathrm{~mL} /$ $\mathrm{min} / 1.73 \mathrm{~m}^{2}$ ) or renal damage (proteinuria or albuminuria) lasting for $\geq 3$ months (Foundation, 2002). However, we defined CKD using a single measurement of the estimated GFR based on the Modification of Diet in Renal Disease equation, which is not influenced by characteristics of the Korean population. Further, we employed the enzymatic method, which might not accurately estimate actual $\mathrm{GFR}^{37}$, to measure creatinine (as opposed to the Jaffe method, which is generally recommended) and tested for proteinuria using a dipstick test. Third, reporting bias might have been present, because the data from cancer survivors were collected through self-report questionnaires. Fourth, there is no information on cancer type among cancer survivors, which makes it impossible to specify risk according to cancer type. Despite these limitations, to our knowledge, this is the first study to examine the features of the association between CKD and cancer survival using national data in Korea. Moreover, using a weighted method adjusting for multiple variables, the ability to generalize the outcome is a strength of our study.

In conclusion, our study demonstrated that cancer survivors appear to be at greater risk for CKD among Korean adults using nationally representative data. Prospective studies are necessary to establish more reliable, strategic, and practical plans for the prevention of CKD in cancer survivors and to minimize the burden on public health in the future.

\section{Acknowledgements}

We thank the members of the Korea Institute for Health and Social Affairs who conducted the national survey and everyone who contributed to this project.

\section{References}

Airy M, Raghavan R, Truong LD, et al (2013). Tubulointerstitial nephritis and cancer chemotherapy: update on a neglected clinical entity. Nephrol Dial Transplant, 28, 2502-9.

Athyros VG, Katsiki N, Doumas M, et al (2013). Effect of tobacco smoking and smoking cessation on plasma lipoproteins and associated major cardiovascular risk factors: a narrative review. Curr Med Res Opin, 29, 1263-74.

Cengiz K (2002). Increased incidence of neoplasia in chronic renal failure (20-year experience). Int Urol Nephrol, 33, 121-6.

Centers for Disease Control and Prevention (CDC) (2011). Cancer survivors-United States, 2007. MMWR. Morbidity and mortality weekly report, $\mathbf{6 0}, 269-72$.

Dekkers IA, Blijdorp K, Cransberg K, et al (2013). Long-term nephrotoxicity in adult survivors of childhood cancer. Clin $J$ Am Soc Nephrol, 8, 922-9.

Demark-Wahnefried W, Aziz NM, Rowland JH, et al (2005). Riding the crest of the teachable moment: promoting longterm health after the diagnosis of cancer. J Clin Oncol, 23, 5814-30.

Denlinger CS, Carlson RW, Are M, et al (2014). Survivorship: introduction and definition. Clinical practice guidelines in oncology. J Natl Compr Canc Netw, 12, 34-45.

Duffy SA, Terrell JE, Valenstein M, et al (2002). Effect of smoking, alcohol, and depression on the quality of life of head and neck cancer patients. Gen Hosp Psychiatry, 24, $140-7$.

Fedirko V, Tramacere I, Bagnardi V, et al (2011). Alcohol drinking and colorectal cancer risk: an overall and doseresponse meta-analysis of published studies. Ann Oncol, 22, 1958-72.

Foundation NK (2002). K/DOQI clinical practice guidelines for chronic kidney disease: evaluation, classification, and stratification. Am J Kidney Dis, 39, 1-266.

Grewal P, Viswanathen VA (2012). Liver cancer and alcohol. Clin Liver Dis, 16, 839-50.

Gritz ER, Nisenbaum R, Elashoff RE, et al (1991). Smoking behavior following diagnosis in patients with stage I nonsmall cell lung cancer. Cancer Causes Control, 2,105-12.

Hounshell J, Tomori C, Newlin R, et al (2001). Changes in finances, insurance, employment, and lifestyle among persons diagnosed with hairy cell leukemia. Oncologist, 6, 435-40

Imai E, Horio M, Nitta K, et al (2007). Estimation of glomerular filtration rate by the MDRD study equation modified for Japanese patients with chronic kidney disease. Clin Exp Nephrol, 11, 41-50.

Jha V, Garcia-Garcia G, Iseki K, et al (2013). Chronic kidney disease: global dimension and perspectives. Lancet, 382, 260-72.

Johansen KL (2007). Exercise in the end-stage renal disease population. J Am Soc Nephrol, 18, 1845-54.

Jorgensen L, Heuch I, Jenssen T, et al (2008). Association of albuminuria and cancer incidence. J Am Soc Nephrol, 19, 992-8.

Levey AS, Bosch JP, Lewis JB, et al (1999). A more accurate method to estimate glomerular filtration rate from serum creatinine: a new prediction equation. Modification of Diet in Renal Disease Study Group. Ann Intern Med, 130, 461-70.

Levey AS, Eckardt KU, Tsukamoto Y, et al (2005). Definition and classification of chronic kidney disease: a position statement from kidney disease: improving global outcomes (KDIGO). Kidney Int, 67, 2089-100.

Liu Y, Hu F, Li D, et al (2011). Does physical activity reduce the risk of prostate cancer? A systematic review and metaanalysis. Eur Urol, 60, 1029-44.

Maisonneuve P, Agodoa L, Gellert R, et al (1999). Cancer in patients on dialysis for end-stage renal disease: an international collaborative study. Lancet, 354, 93-9.

Maskarinec G, Murphy S, Shumay DM, et al (2001). Dietary changes among cancer survivors. Eur J Cancer Care, 10,12-20.

Ministry of health and welfare, N.C.C. Cancer Fact and Figures 2012 in the republic of Korea

Ogle KS, Swanson GM, Woods N, et al (2000). Cancer and comorbidity: redefining chronic diseases. Cancer, 88, 653-63.

Quarmby S, Kumar P, Kumar S (1999). Radiation-induced normal tissue injury: role of adhesion molecules in leukocyte-endothelial cell interactions. Int J Cancer, 82 , 385-95.

Siddik ZH (2005). Mechanisms of action of cancer chemotherapeutic agents: DNA-Interactive alkylating agents and antitumour platinum-based drugs. Cancer Handbook, Wiley, New Jersey.

Sprague BL, Trentham-Dietz A, Nichols HB, et al (2010). Change in lifestyle behaviors and medication use after a diagnosis of ductal carcinoma in situ. Breast Cancer Res Treat, 124, 487-95.

Steele MR, Belostotsky V, Lau KK (2012). The dangers of substance abuse in adolescents with chronic kidney disease: 
a review of the literature. Cannt $J, 22,15-22$.

Stump CS (2011). Physical activity in the prevention of chronic kidney disease. Cardiorenal Med, 1, 164-73.

Thomas F Babor, John CH-B, J BS, et al (2001). AUDIT, the alcohol use disorders identification test : guidelines for use in primary care. World Health Organization, Dept. of mental health and substance dependence, Geneva, Switzerland.

Vajdic CM, McDonald SP, McCredie MR, et al (2006). Cancer incidence before and after kidney transplantation. JAMA, 296, 2823-31.

White SL, Polkinghorne KR, Cass A, et al (2009). Alcohol consumption and 5-year onset of chronic kidney disease: the AusDiab study. Nephrol Dial Transplant, 24, 2464-72.

Willi C, Bodenmann P, Ghali WA, et al (2007). Active smoking and the risk of type 2 diabetes: a systematic review and meta-analysis. JAMA, 298, 2654-64.

Wolin KY, Yan Y, Colditz GA, et al (2009). Physical activity and colon cancer prevention: a meta-analysis. Br J Cancer, 100, 611-6.

Wu Y, Zhang D, Kang S (2013). Physical activity and risk of breast cancer: a meta-analysis of prospective studies. Breast Cancer Res Treat, 137, 869-82.

Xin X, He J, Frontini MG, et al (2001). Effects of alcohol reduction on blood pressure: a meta-analysis of randomized controlled trials. Hypertension, 38, 1112-7.

Yao X, Panichpisal K, Kurtzman N, et al (2007). Cisplatin nephrotoxicity: a review. Am J Med Sci, 334, 115-24. 